\title{
Combinación de Osteotecnia más Conservación de Músculos en Montaje Único de Canis lupus familiaris
}

\author{
Combination of Osteotecnia and Preservation of Muscles \\ in Unique Assembly of Canis lupus familiaris
}

\author{
Mauricio Armando Villarroel Guerra ${ }^{1}$ \& Nazareth Andrea Troncoso Felipe ${ }^{2}$
}

VILLARROEL, G. M.A.\& TRONCOSO, F. N. A. Combinación de osteotecnia más conservación de músculos en montaje único de Canis lupus familiaris. Int. J. Morphol., 35(1):351-356, 2017.

RESUMEN: Para la enseñanza de la Anatomía siempre se está en búsqueda de alternativas para la conservación y presentación de preparados anatómicos, realizando variaciones de diferentes técnicas, que permitan presentar especímenes con fines didácticos, para lograr un mejor aprendizaje, comprensión y motivación por la Morfología. El objetivo de este trabajo fue utilizar una combinación de osteotecnia más la conservación de músculos, en un montaje único que permite estudiar en una mitad el esqueleto, y en la otra, la musculatura superficial de un Canis lupus familiaris. Se utilizó un cadáver del especimen fijado por congelación, luego se realizó una limpieza general, extracción de vísceras y retiro de tegumentos previo al corte sagital. En la mitad derecha se realizó la limpieza de los huesos mediante ebullición combinada con métodos mecánicos y posteriormente, el desengrasado y blanqueado de ellos previo al ensamble del esqueleto. Para la conservación de músculos la mitad izquierda del cuerpo fue sumergida en "solución fijadora conservadora chilena" libre de formol, por 31 días, tras lo cual se realizó la disección de fascias y tejido graso para delimitar músculos superficiales. Una vez tratado cada segmento, se procedió al montaje combinado de las mitades en posición anatómica del espécimen, usando suturas desde craneal a caudal, sobre pedestales. La técnica utilizada de montaje combinado proporciona un material anatómico-didáctico flexible, de bajo costo y escasa toxicidad, con la potencialidad de permitir reforzar de manera interactiva el aprendizaje significativo de los estudiantes, facilitando la observación y estudio de la topografía de huesos y músculos.

PALABRAS CLAVE: Técnica anatómica; Osteotecnia; Conservación de músculos; Especímenes anatómicos.

\section{INTRODUCCIÓN}

El desarrollo de piezas anatómicas que faciliten la adquisición y comprensión de las diferentes estructuras que componen un especimen no sólo es ideal, sino que resulta una necesidad concreta para la enseñanza de la Morfología (Villarroel \& Medina, 2011; Lizana et al., 2015). Por ello, la búsqueda de técnicas que puedan conservar mejor especímenes anatómicos u órganos, implicando un menor costo y además, que los reactivos que sean utilizados no sean dañinos e invasivos para la salud humana, es un estímulo constante en el ámbito anatómico.

Se entiende por osteotecnia al conjunto de procedimientos naturales, químicos y/o físicos, a través del cual se puede tratar un especimen con el fin de obtener un hueso, segmento o esqueletos limpios, sin presencia de residuos orgánicos, facilitando la observación de formaciones anatómicas, con el fin de apreciar la posición exacta en que están dispuestos los huesos en el organismo y ser usado con fines de investigación, educativos o para ser exhibidos dentro de una colección (Cañete Betancourt et al., 2014).

Los pilares fundamentales para la elaboración de muestras anatómicas que conserven su aspecto semejante a su estado fresco, son las técnicas de fijación, conservación y preservación (Bustamante et al., 2007). Estas técnicas han sido relevantes para el estudio de la anatomía humana y veterinaria, ya que permiten mantener especímenes u órganos protegidos contra su deterioro y facilitar su uso con fines didácticos, académicos y de investigación (Wolff et al., 2012).

La disminución en el uso de soluciones fijadoras y/o conservadoras en base a formol, ha implicado una reducción de riesgos para los estudiantes, docentes y auxiliares y así evitar efectos secundarios de irritación, toxicidad e incluso cáncer, atribuidos a dicho compuesto (WHO, 2010), además de implementar el uso de técnicas de trabajo que dismi- 
nuyan su incidencia sobre el ambiente (Muñetón Gómez \& Ortiz, 2011). Para ello, se han creado o modificado técnicas que sustituyen la formalina por otros compuestos con menos efectos adversos a la salud, como son las soluciones a base de glicerina, que son capaces de conservar el volumen y consistencia blanda en las muestras anatómicas (Hammer et al., 2012; Correa Alarcon, 2005).

Dado que la enseñanza de la morfología macroscópica en las instituciones se ve enfrentada, en algunas ocasiones, a las dificultades de obtención de especímenes anatómicos como material didáctico, es de suma importancia el conocimiento de la preservación y restauración de preparados anatómicos presentes en los laboratorios, utilizando técnicas accesibles y con bajo costo (Rodríguez Palomo \& Ramírez Zamora, 2009).

El objetivo de este trabajo fue utilizar la osteotecnia y la conservación de músculos, para presentar en un especimen anatómico de Canis lupus familiaris, un montaje único de ambas técnicas que permitiera observar y estudiar en una mitad del especimen el esqueleto, y en la otra, su musculatura superficial y flexibilidad de las articulaciones.

\section{MATERIAL Y MÉTODO}

El presente trabajo se realizó en el Laboratorio de Anatomía de la Facultad de Ciencias Naturales y Exactas de la Universidad de Playa Ancha en Valparaíso, Chile. Se utilizó un cadáver de canino (Canis lupus familiaris) donado al laboratorio. El especimen macho adulto, de 18 a 24 meses, presentaba contusiones musculares a nivel torácico y pélvico del lado derecho y fue fijado por congelación a $-15^{\circ} \mathrm{C}$.

La metodología fue dividida en 3 fases: La fase I se refirió a la osteotecnia, la fase II consistió en la conservación de musculatura y la fase III correspondió al montaje combinado de ambas técnicas. En cada una de las fases hubo registro fotográfico utilizando cámara Canon EOS Rebel T3i.

Fase I. Se realizó en cuatro etapas:

a) Preparación: Consistió en lavado general del especimen y la extracción de los órganos internos. Posteriormente, se realizaron sucesivos lavados para eliminar remanentes de residuos orgánicos. Luego fueron retirados los tegumentos, exceptuando las regiones: oral, de la nariz, orbitaria, auricular, región palmar y plantar. Se disecó la musculatura desde craneal a caudal del lado derecho del especimen. Para finalizar, se realizó un corte sagital a nivel de los procesos transversos izquierdos de las vértebras, dividiendo al especimen en dos secciones, la mitad izquierda fue utilizada en la fase II. b) Limpieza de los huesos: Primero se desarticularon los esqueletos axial y apendicular. Luego, cada hueso fue sometido a ebullición en agua corriente con óxido de calcio $50 \mathrm{~g} / \mathrm{L}$ para acelerar el proceso de desprendimiento de tejidos remanentes. Por último, cada pieza ósea fue lavada, secada a temperatura ambiente y guardada individualmente.

c) Desengrasado y blanqueamiento: El protocolo utilizado consistió en sumergir los huesos en hexano por 90 minutos para retirar restos de grasa; luego, lavados en agua corriente y posteriormente fueron secados a temperatura ambiente. El blanqueamiento de los huesos se consiguió con $\mathrm{H}_{2} \mathrm{O}_{2}$ al 30 $\%$, en los tiempos fueron variables entre 1-3 horas, en función de la clasificación de los huesos (largos, cortos, planos o irregulares) y con variaciones respecto a su tamaño y grosor.

d) Ensamblaje del esqueleto: Con la finalidad de unir el cráneo con la columna vertebral, se introdujo un alambre galvanizado de $2 \mathrm{~mm}$ trenzado, revestido por un tubo de PVC transparente de $8 \mathrm{~mm}$, por el canal vertebral. El resto de los huesos se ensambló insertando hilo de nylon blanco de $0,8 \mathrm{~mm}$, a través de pequeñas perforaciones realizadas en los huesos utilizando la herramienta multipropósito DREMEL $^{\circledR}$ con broca de $1 \mathrm{~mm}$, respetando la anatomía del especimen (Fig. 1).

Fase II. Para conservar la musculatura del preparado, se aplicó la "solución fijadora conservadora chilena (sfcCh)" utilizada en este tipo de procedimientos (Rojas \& Ruiz, 2010; Franco, 2014; Ortega, 2014; Ballesteros et al., 2016), la cual se compone de cloruro de sodio, nitrato de sodio, glicerina, alcohol etílico, cloruro de benzalconio, formaldehido y esencia de eucalipto. Esta técnica fue utilizada modificada de la original reemplazando el formaldehido por un litro de alcohol etílico. (Se sugiere para profundizar en la composición y fórmula de sfcCh revisar a Ortega).

La mitad izquierda resultante de la etapa de preparación de la Fase I, fue sumergida en un contenedor cerrado, a temperatura ambiente $\left(20 \pm 5^{\circ} \mathrm{C}\right)$, con la solución cubriendo totalmente al especimen y mantenida durante 4 días. Posteriormente, se reemplazó completamente la solución conservadora, permaneciendo 27 días bajo las mismas condiciones anteriormente señaladas. Transcurrido el tiempo, se extrajo el especimen de la solución.

Empleando material de disección ad hoc, se inició el proceso de retiro de tejido adiposo y fascias, luego se realizó la delimitación de cada músculo superficial facilitando su identificación. En esta etapa se trabajó a temperatura ambiente, procurando mantener la humedad del preparado anatómico, rociando solución fijadora conservadora con un pulverizador manual, después de cada sesión de una hora de trabajo. 
Fase III. Al tener finalizados los tratamientos de las dos mitades resultantes de las fases I y II, se continuó con el ensamble de las secciones. Se unieron las secciones procurando en todo momento la coincidencia de todas las formaciones anatómicas y, de manera manual, se suturó intercaladamente, desde craneal a caudal, utilizando hilo de nylon blanco de $0,20 \mathrm{~mm}$.

Posteriormente, se realizó el montaje del especimen en una base de madera con cuatro pedestales que mantuvieron al canino por sus regiones cervical, torácica, pélvica y miembro torácico izquierdo.

Para mantener y preservar la musculatura del preparado, se aplicó cada dos semanas, la misma solución fijadora por aspersión, utilizando un difusor manual, evitando la llegada directa de luz solar.

\section{RESULTADOS}

La utilización de la técnica de osteotecnia y conservación de músculos en montaje combinado, permitió obtener un preparado anatómico de piezas reales y de duración prolongada.

Los materiales utilizados en ambas técnicas permitieron una fácil manipulación del especimen, el cual no emite olores desagradables ni nocivos para la salud. El ensamble de los huesos de miembros no presentó inconvenientes para movilidad y otorgó la resistencia necesaria para su manipulación. Tanto músculos superficiales, como huesos y sus estructuras pudieron ser rotulados para facilitar su observación y comprensión.

Si se analiza en forma particular los resultados de cada técnica, se observa con respecto a la osteotecnia que a través de la metodología utilizada, se obtuvo el blanqueamiento esperado en cada pieza ósea (Fig. 1). Por otra parte, el hecho de tomar en consideración los tiempos de reacción para huesos de acuerdo a los criterios señalados en la metodología, permitió evitar la descalcificación. El ensamble de los huesos del esqueleto axial con alambre, fue adecuado, ya que permitió articular inmediatamente con el cráneo, procurando dar forma a las curvaturas naturales del especimen (Fig. 2).

En los resultados de Fase II se observó que la mitad izquierda tratada con la "sfcCh" modificada sin formol, mostró buenos resultados, manteniendo una coloración y textura similares al estado fresco, libre de olor desagradable o irritante y sin crecimiento de microorganismos ni

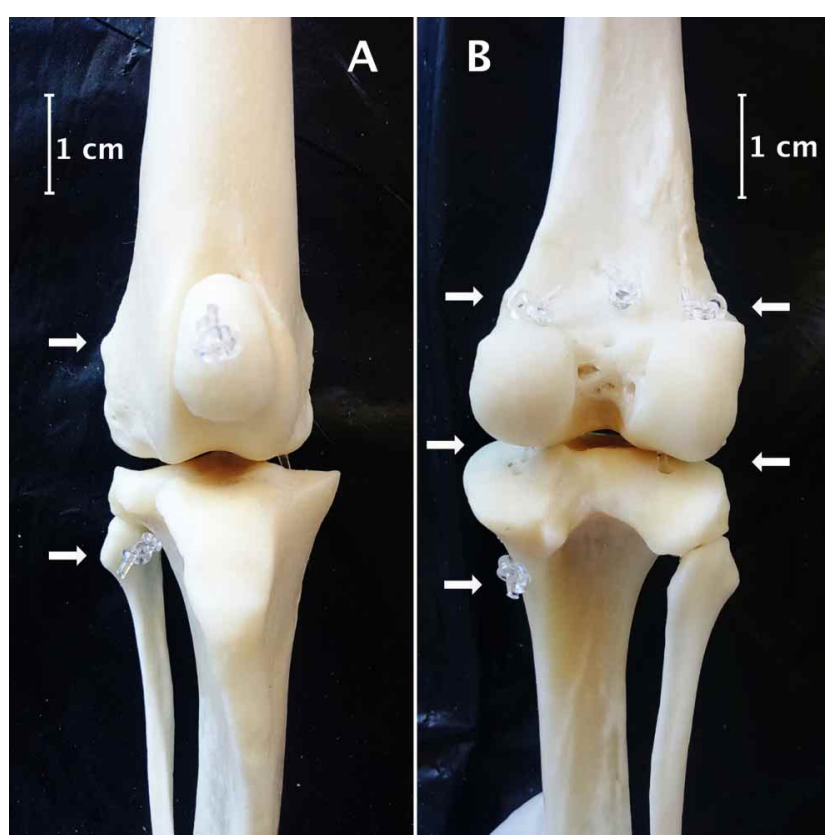

Fig. 1. Resultado final Fase I blanqueamiento y ensamble de articulación de rodilla derecha de Canis lupus familiaris. Las flechas señalan puntos donde se ubican nudos y/o paso de hilo nylon utilizado para ensamblar los huesos. Vista anterior (A) y posterior (B).

descomposición observables a ojo desnudo, además conservó la flexibilidad articular requerida para el montaje combinado (Fig. 3). De igual manera, la solución mantuvo la mayoría de características del pelaje en los lugares donde no fue disecado (Fig. 4).

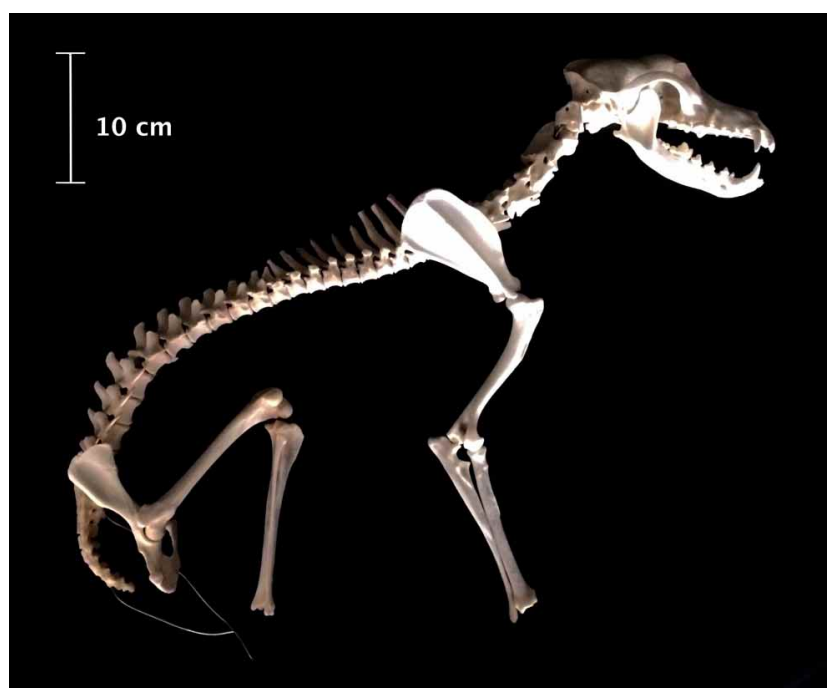

Fig. 2. Ensamble de mitad derecha de columna vertebral y cráneo de Canis lupus familiaris, utilizando alambre que cruza a través del canal vertebral. Los huesos de los miembros torácico y pélvico se presentan superpuestos previos a su ensamble. Vista lateral derecha. 


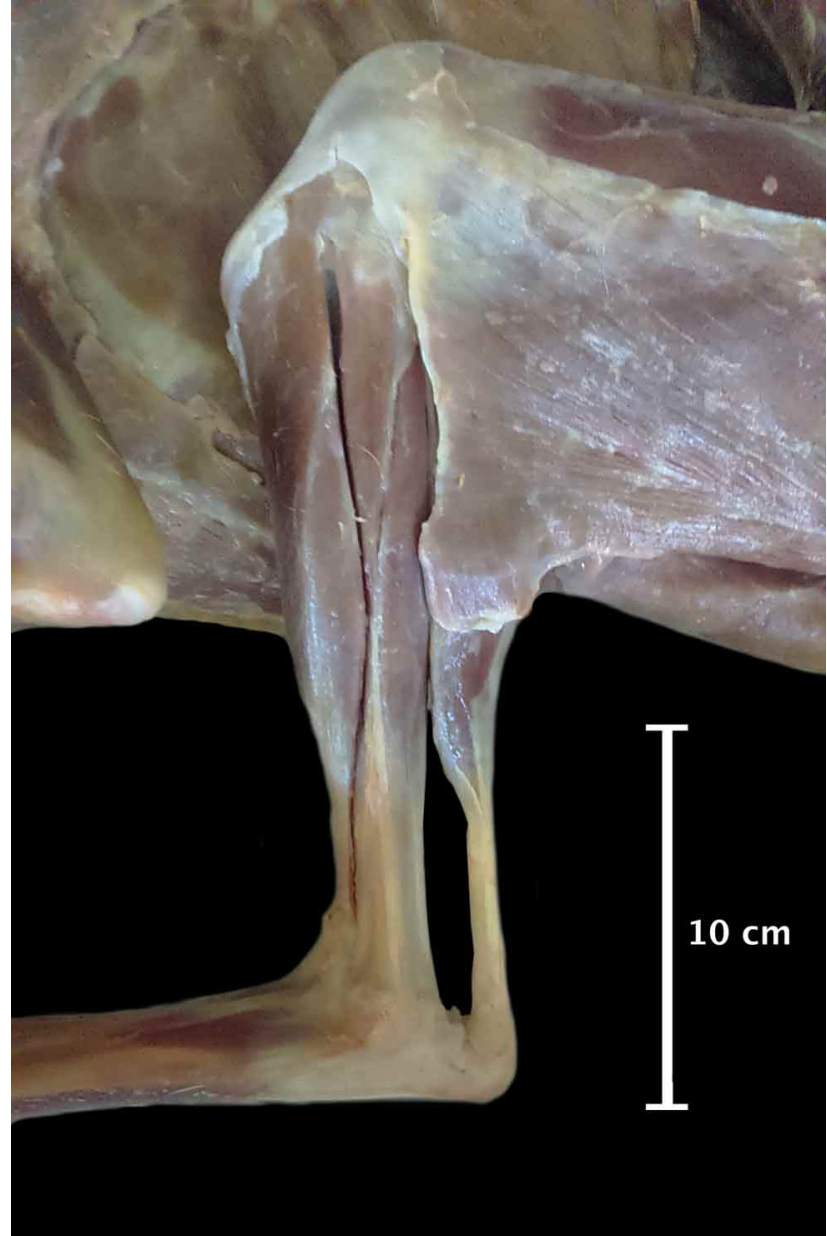

Fig. 3. Conservación de miembro pélvico izquierdo de Canis lupus familiaris, con delimitación de parte de la musculatura. (Fotografía capturada 40 días después de la primera inmersión). Vista lateral izquierda.

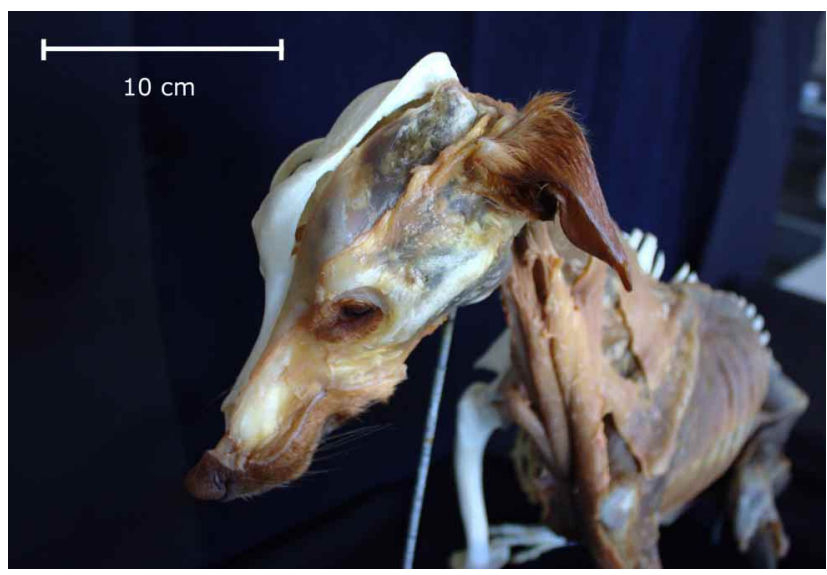

Fig. 4. Presentación de las características del pelaje a nivel de las regiones auricular, orbitaria, oral y de la nariz, en mitad izquierda del preparado. Vista craneal (antero-lateral izquierda) del preparado anatómico de Canis lupus familiaris montaje combinado de osteotecnia y conservación de músculos. (Fotografía capturada 180 días después del montaje combinado).
Como resultado final del montaje combinado de ambas técnicas (Fase III), las sucesivas suturas realizadas a nivel de las vértebras dieron la firmeza necesaria a la muestra, sin mayores limitaciones de movilidad del preparado. La utilización de pedestales que sujetan al especimen en las regiones cervical, torácica, pélvica y miembro torácico izquierdo ayudaron a mantener una postura similar a la natural, del especimen, favoreciendo la estabilidad y presentación general de la muestra (Figs. 5, 6 y 7).

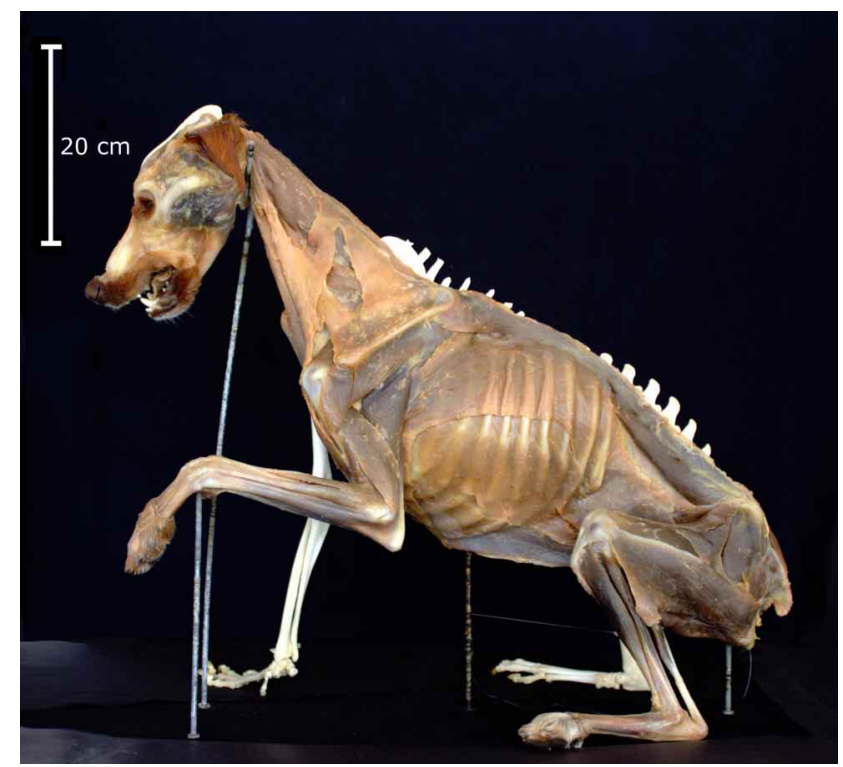

Fig. 5. Presentación de musculatura del preparado anatómico de Canis lupus familiaris montaje combinado de osteotecnia y conservación de músculos. (Fotografía capturada 180 días después del montaje combinado). Vista lateral izquierda.

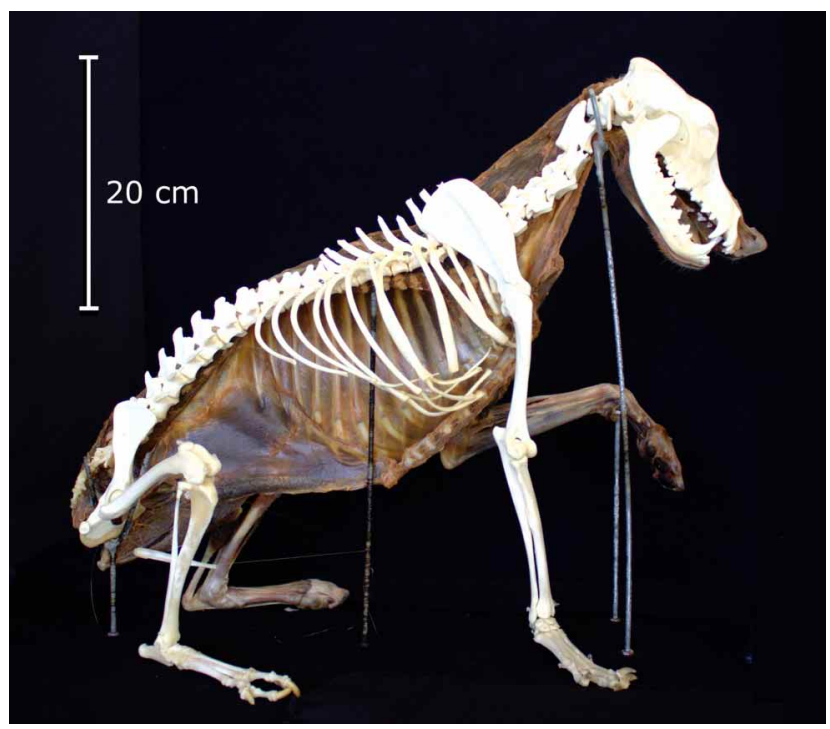

Fig. 6. Presentación del esqueleto del preparado anatómico de Canis lupus familiaris montaje combinado de osteotecnia y conservación de músculos. (Fotografía capturada 180 días después del montaje combinado). Vista lateral derecha. 

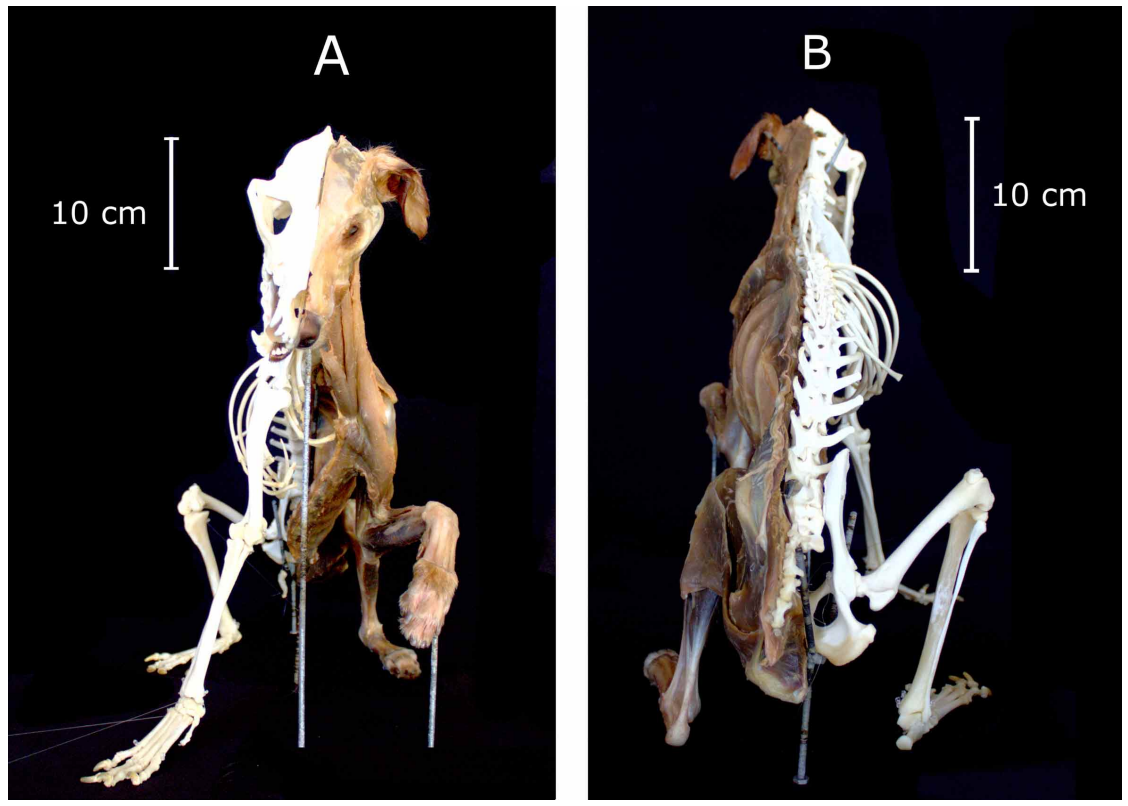

Fig. 7. Vistas craneal (A) y caudal (B) de preparado anatómico de Canis lupus familiaris montaje combinado de osteotecnia y conservación de músculos. (Fotografía capturada 180 días después del montaje combinado).

\section{DISCUSIÓN}

Gran parte del éxito de la osteotecnia y su etapa de blanqueado radica en que permite observar periódicamente los huesos, ya que cuando adquieren el color deseado, deben ser sacados de la solución, lavados y secados a temperatura ambiente. Lo anterior, evita la descalcificación excesiva, coincidiendo con lo señalado por Cañete Betancourt et al. Trabajos pre-experimentales realizados por los autores, indican que el tiempo de exposición de los huesos durante el uso de la técnica de blanqueado variará dependiendo de la especie, su edad y la clasificación y tamaño del hueso tratado.

En la etapa del ensamblado del esqueleto, los materiales para unir cada pieza ósea fueron, a nuestro juicio, los adecuados, ya que permitieron unión y flexibilidad de movimientos en las diferentes articulaciones.

El tratamiento realizado a la mitad izquierda del cuerpo del Canis lupus familiaris, para conservar su musculatura, impidió el crecimiento de microorganismos y la descomposición, a ojo desnudo y además, mantuvo la flexibilidad articular, permitiendo diferenciar claramente entre los diferentes músculos y sus tendones, coincidiendo con los resultados de otras técnicas que utilizaron soluciones exentas de formol (Hammer et al., 2011) y Correa Alarcon, quienes utilizaron muestras humanas, y cuyos resultados fueron similares al desarrollado en nuestro trabajo.
La utilización "sfcCh" para preservar la musculatura de especímenes, con la variación de no incluir formol, mostró ser efectiva y eficiente para la conservación del material biológico, ya que se evita el alto grado de rigidez tanto de musculatura como de articulaciones y pérdida de color natural (Rivera $e t$ al., 2009). De igual forma, los efectos irritantes se ven eliminados dado a la baja toxicidad de sus componentes. En cuanto a la mantención para preservar la musculatura, no hay necesidad de sumergir nuevamente en cubeta con solución para la conservación, ya que se aplica solución por aspersión aun cuando esto requiere observar la pieza regularmente una vez por semana.

Aunque en la enseñanza de la anatomía el uso de preparaciones cadavéricas fijadas y conservadas en soluciones a base de formol, sigue siendo utilizada (Bustamante et al.) siempre será un aporte implementar y modificar algunas de las técnicas utilizadas en otros ámbitos (Muñetón Gómez \& Ortiz, 2012; Muñetón Gómez \& Ortiz, 2013) para la conservación de preparados anatómicos.

Coincidimos con Rivera et al. (2014) al señalar que entre las diferentes técnicas anatómicas que existen en la enseñanza de la anatomía, cada una puede presentar ventajas y desventajas, las cuales deben ser analizadas al momento de incluir su uso en cualquier laboratorio de morfología, de acuerdo a los objetivos planteados, siendo lo ideal utilizar una combinación de diferentes técnicas con la finalidad de abarcar la anatomía desde un todo, pero sin descuidar la calidad de los preparados obtenidos.

La utilización de la técnica de osteotecnia y conservación de músculos en montaje combinado ha permitido obtener un preparado anatómico que, se espera, pueda ser replicado y que los resultados permitan ser evaluados en mayor tiempo.

Se debe destacar que dentro de cada técnica y su montaje combinado, se requiere un análisis morfoestructural de las piezas en todas las etapas del proceso, lo cual enriquece la técnica y la calidad de la muestra.

Las técnicas utilizadas en un montaje combinado son aplicados por primera vez en el Laboratorio de Anatomía de la Facultad de Ciencias Naturales y Exactas de la Universidad de Playa Ancha, proporcionando un material anatómico-di- 
dáctico que combina una técnica seca y otra húmeda, con la finalidad de conocer la anatomía del Canis lupus familiaris, muestra que podría ser utilizada por un largo tiempo, contribuyendo potencialmente a reforzar de manera interactiva el aprendizaje significativo de los estudiantes, al facilitar la observación topográfica de huesos y músculos. Finalmente, es necesario destacar que la técnica utilizada es de bajos costo y toxicidad.

AGRADECIMIENTOS. Al Dr. Pablo Lizana Arce y al Dr. Mauricio Valenzuela Harrington por sus críticas y aportes al presente trabajo. A los Sres. Enrique Flores y Elías Arredondo por su apoyo técnico.

VILLARROEL, G. M. A. \& TRONCOSO, F. N. A. Combination of osteotecnia and preservation of muscles in unique assembly of Canis lupus familiaris. Int. J. Morphol., 35(1):351-356, 2017.

SUMMARY: The teaching of anatomy is always in search of alternatives for the preservation and presentation of anatomical preparations, doing variations of different techniques that allow to present specimens with didactic goals so as to get a better learning, comprehension and motivation for morphology. The purpose of this study was to use a combination of osteotecnia and the preservations of muscles in a unique assembly allowing to study half of the skeleton and, in the other, the superficial muscles of a Canis lupus familiaris. A corpse of the specimen was used, fixed by freezing; then, a general cleaning was carried out, extraction of viscera and removal of teguments before the sagittal cut. On the right half, using boiling combined with mechanical methods and, subsequently, their degreasing and bleaching, prior to the skeleton's assembly carried out the cleaning of the bones. For the preservation of muscles, the left half of the body was submerged in a "Chilean preserving and fixative solution" free from formaldehyde for 31 days, following the dissection of fascia and fatty tissue to delimit superficial muscles. Once each segment is treated, the combined assembly of the halves is made in anatomical position of the specimen, using stitches from cranial to caudal, over pedestals. The utilized technique of combined assembly provides a flexible didactic-anatomical material, of low-cost and low-toxicity, with the potentiality to allow reinforcing in an interactive way the meaningful learning of the students, making the observation easier as well as the study of topography of bones and muscles.

KEY WORDS: Anatomical technique; Osteotecnia; Conservation of muscles; Specimens anatomical.

\section{REFERENCIAS BIBLIOGRÁFICAS}

Ballesteros, L. E.; Estupiñan, H. Y. \& Ballesteros, D. R. Vascular variations in urinary tract and abdominal cavity. Case report. Int. J. Morphol., 34(1):4049, 2016.

Bustamante, A. M. F.; Prieto, G. R. H. \& Binvignat Gutiérrez, Octavio. Preservation of human placenta. Anatomical technique. Int. J. Morphol., 25(3):545-8, 2007.

Cañete Betancourt, G.; Sánchez Pellitero, J. M. \& Noda Cuellar, L. Ensamblaje artesanal de un esqueleto canino mediantes variantes de la osteotecnia. REDVET Rev. Electron. Vet., 15(9):1-15, 2014.
Correa Alarcon, F. Conservación de piezas anatómicas en seco mediante el método de prives. REDVET Rev. Electron. Vet., 6(5):1-8, 2005.

Franco, J. Restauración de piezas anatómicas humanas. Tesis de Magister. Bogotá, Universidad Nacional de Colombia, 2014.

Hammer, N.; Löffler, S.; Feja, C.; Bechmann, I. \& Steinke, H. Substitution of formaldehyde in cross anatomy is possible. J. Natl. Cancer Inst., 103(7):610-1, 2011.

Hammer, N.; Löffler, S.; Feja, C.; Sandrock, M.; Schmidt, W.; Bechmann, I. \& Steinke, H. Ethanol-glycerin fixation with thymol conservation: a potential alternative to formaldehyde and phenol embalming. Anat. Sci. Educ., 5(4):225-33, 2012.

Lizana, P. A.; Merino, C.; Bassaber, A; Henríquez, R.; Vega-Fernández, G. \& Binvignat, O. Learning human anatomy using three-dimensional models made from real-scale bone pieces: experience with the knee joint among pre-service biology teachers. Int. J. Morphol., 33(4):1299-306, 2015.

Muñetón Gómez, C. A. \& Ortiz, J. A. Preparación en glicerina: una técnica para la conservación prolongada de cuerpos en anatomía veterinaria. Rev. Med. Vet., (26):115-22, 2013.

Muñetón Gómez, C. A. \& Ortiz, J. A. Plastinación: un instrumento complementario para el desarrollo del proceso enseñanza-aprendizaje de la anatomía. Rev. Med. Vet., (23):111-7, 2012.

Muñetón Gómez, C. A. \& Ortiz, J. A. Conservación y elaboración de piezas anatómicas con sustancias diferentes al formol en la Facultad de Ciencias Agropecuarias de la Universidad de La Salle. Rev. Med. Vet., (22):51-5, 2011.

Ortega, L. Recuperación restauración de componentes anatómicos humanos extremidades superiores. Tesis de Magister. Bogotá, Universidad Nacional de Colombia, 2014.

Rivera, D. M. L.; Suárez, R. C. J.; Yate, V. A.; Cruz, M. C. E.; Barahona, B. G. S.; Cortes, N. A. X. \& Arias, L. L. A. Comparación de técnicas de conservación morfológica y su posible aplicación para la enseñanza de la anatomía. Morfolia, 6(3), 2014.

Rivera, M. C.; Bonino, F.; Fioretti, C.; Galán, M.; Gigena, S.; Moine, R.; Mouguelar, H.; Natali, J. \& Quinteros, R. Multivaried analysis applied at the stage of dehydration in the plastination technique in horse's kidney. Int. J. Morphol., 27(3):855-9, 2009.

Rodríguez Palomo, D. \& Ramírez Zamora, J. Técnica de conservación de huesos en peróxido de hidrógeno. Med. Leg. Costa Rica, 26(2):117-23, 2009.

Rojas, O. J. D. \& Ruiz, D. S. S. Empleo de solución fijadora conservadora chilena como alternativa al uso del formaldehido para la preservación de tejidos. En: V Congreso Colombiano de Morfología. Cali - Colombia, Noviembre de 2009. Int. J. Morphol., 28(1):337-40, 2010.

Villarroel, G. M. \& Medina, O. E. Anatomical technique to restore and/or reproduce human bone pieces difficult to obtain for research and teaching science. Int. J. Morphol., 29(2):532-6, 2011.

Wolff, D.; Villa, P.; Neirreitter, A.; Ruibal, C.; Ugon, G. A.; Salgado, G. \& Cantín, M. Comparative study between conservative solutions with and without formaldehyde in human placenta. Int. J. Morphol., 30(2):432-8, 2012.

World Health Organization (WHO). WHO Guidelines for Indoor Air Quality: Selected Pollutants. Génova, World Health Organization, 2010.

Dirección para correspondencia:

Dr. Mauricio Villarroel Guerra

Departamento Disciplinario de Biología

Facultad de Ciencias Naturales y Exactas

Universidad de Playa Ancha

Casilla 34-V

Valparaíso

CHILE

Email: mvillarr@upla.cl

Recibido : 07-09-2016

Aceptado: 25-10-2016 